\title{
HEARTBURN IN LATE PREGNANCY. MANOMETRIC STUDIES OF ESOPHAGEAL MOTOR FUNCTION *
}

\author{
By RICHARD NAGLER AND HOWARD M. SPIRO \\ (From the Department of Internal Medicine, Yale University School of Medicine, \\ New Haven, Conn.)
}

(Submitted for publication May 27, 1960; accepted January 13, 1961)

Heartburn is a burning, often hot sensation which may localize in the throat or substernal region. It is especially common during pregnancy. Gastroesophageal reflux and neuromuscular dysfunction of the esophagus have been suggested as underlying pathophysiological mechanisms, but contradictory evidence has prevented a clear definition of the cause of heartburn.

A prospective clinical study of 560 pregnant women disclosed that 50 per cent developed heartburn which invariably disappeared after delivery (1). The self-limited character of pregnancy makes it an ideal experimental period during which to study heartburn. Transducer pressure recordings of esophageal motility are well adapted to such a study.

This paper reports the results of serial esophageal motility studies in pregnant women with and without heartburn and in a group of asymptomatic normal women of similar age.

\section{METHODS}

Subjects. Fifty-seven esophageal motility studies were performed on 20 pregnant women who complained of heartburn during pregnancy. The 8 primiparae and 12 multiparae ranged in age from 16 to 39 years, with an average age of 28 . Similarly, 63 recordings were obtained from 19 pregnant women who never developed heartburn throughout pregnancy; in this group were 10 primiparae and 9 multiparae, 20 to 35 years old, with an average age of 26 . Each women was studied at least once during and after pregnancy, but the total number of studies in each subject depended on the month of pregnancy when the patient was first seen. Fourteen asymptomatic normal nonpregnant women, 22 to 44 years old, with an average age of 26 , served as controls; each subject was studied 6 times at monthly intervals.

Technique. The technique of recording esophageal motility utilizes 3 polyethylene catheters, each with an internal diameter of $1.5 \mathrm{~mm}$, which are fastened together and sealed at the tips. A single lateral opening in each

* Supported in part by United States Public Health Service Grants C2578, A1785, and A3473 and the Norwich Chapter of the Connecticut Heart Association. at 5-cm intervals permits simultaneous recording of pressure changes developing from 3 sites within the esophagus and its sphincters. The catheters are waterfilled and transmit pressures to 3 Sanborn electromanometers, the changes in pressure being recorded on a Sanborn multi-channel direct writing recorder. During every recording the vertical position of the electromanometers is readjusted to the same level as the subject's esophagus. This minimizes any hydrostatic effect and permits consistency in serial recording techniques. Swallowing is signaled by simultaneous recordings of laryngeal movement by means of a specially adapted Sanborn pulse wave attachment placed on the skin adjacent to the thyroid cartilage. A pneumographic belt around the chest indicates respiratory movements.

Patients fast for a minimum of 6 hours prior to examination. The catheters are passed through the mouth into the stomach without the use of a local anesthetic. With the patient supine, they are withdrawn, centimeter by centimeter, while pressure changes are continuously recorded in the resting state, and also following a "dry" swallow and the ingestion of approximately $10 \mathrm{ml}$ of water.

When the tip of the catheter lies below the diaphragm, inspiration produces a positive pressure response which reflects increased intra-abdominal pressure. When the tip is above the diaphragm, the normal decrease in pressure in the thorax on inspiration produces a negative deflection. This point of pressure reversal occurs at the diaphragm and is called the "effective diaphragmatic hiatus." This convenient physiological landmark permits localization of the catheters without the use of X-ray or fluoroscopy.

All records are analyzed in a uniform manner. The zero point is the pressure recorded from the stomach 5 $\mathrm{cm}$ distal to the point of respiratory reversal in each subject. The catheters are held in this position, as much as possible, by a trained technician to avoid any vertical movement. The fundal esophageal pressure gradient is obtained by subtracting the mean resting intra-esophageal pressure from the mean resting fundal or zero pressure. A high pressure zone from 1 to $4 \mathrm{~cm}$ long, extending usually from just below the effective diaphragmatic hiatus to a point 1 to $2 \mathrm{~cm}$ above it, functions as an intrinsic sphincter mechanism and is called the inferior esophageal sphincter or vestibule (2-5). Sphincter tone is recorded as the mean resting end-expiratory pressure, which is represented by the pressure gradient between the fundus of the stomach and the sphincter during expiration. 
The best defined resting pressure recorded during withdrawal of the 3 catheters through the sphincter was selected as representative. Relative to resting sphincter pressure, negative and positive readings in this zone develop in response to deglutition and are believed to represent relaxation and contraction of the sphincter, respectively; these are expressed only in qualitative terms. Primary peristalsis (Figure 2A) is represented by the peak positive pressures produced by the muscular contraction moving down the esophagus in response to swallowing (6-10). Both maximal and mean pressures from the middle third of the esophagus are tabulated. Secondary peristalsis (Figure 1) differs from primary peristalsis only in the mode of initiation; primary peristaltic waves follow deglutition while secondary peristaltic waves are initiated by distention (11-13). The frequency of nonpropulsive responses to swallowing (Figure 2B, C) is expressed as a percentage of the total number of swallows. An effort was made to standardize the number of swallows per recording, but a few individuals were less cooperative and swallowed more often. We have arbitrarily considered nonpropulsive responses to more than 25 per cent of the swallows as abnormal. This is based upon the observation that almost all of our asymptomatic, young, normal control subjects had less than this percentage of nonpropulsive responses to swallowing. Spontaneous nonpropulsive contractions (Figure 2D) are designated as "only a few" (less than 10

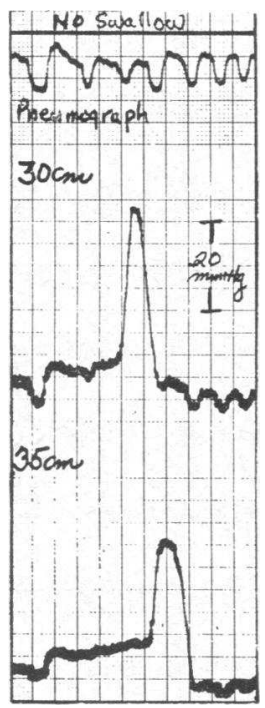

Fig. 1. Electromanometric Recording of a secondARY PERISTALTIC WAVE. This secondary peristaltic wave is propulsive but not preceded by a swallow, which is the only differentiating feature from the primary peristaltic wave that follows swallowing (Figure $2 \mathrm{~A}$ ). The numbers on the left are distances from the incisors. The recording speed used in Figures 1 through 8 was $2.5 \mathrm{~mm}$ per second.
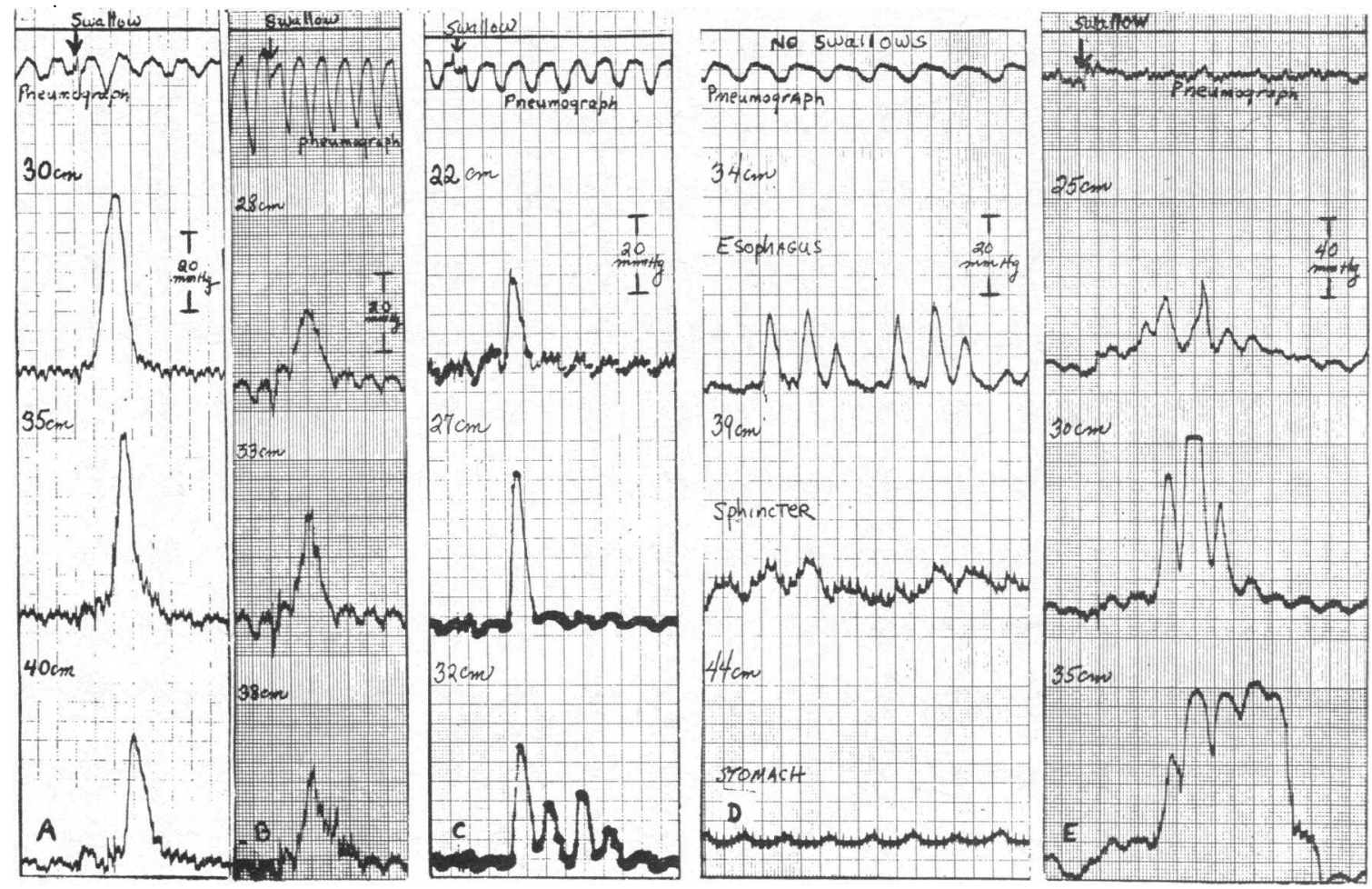

Fig. 2. Typical esophageal Responses of various pregnant women. A) Normal propulsive response to a "dry" swallow; B) nonpropulsive response to a "dry" swallow; C) nonpropulsive repetitive response to a "dry" swallow; D) nonpropulsive repetitive response not preceded by a swallow; E) diffuse esophageal spasm after a "dry" swallow. The numbers on the left of each panel are distances from the incisors. 
TABLE I

Control subjects *

\begin{tabular}{|c|c|c|c|c|c|c|c|c|}
\hline \multirow[b]{2}{*}{ Subject } & \multirow[b]{2}{*}{ Age } & \multirow{2}{*}{$\begin{array}{l}\text { Study } \\
\text { no. }\end{array}$} & \multirow{2}{*}{$\begin{array}{l}\text { Fundal } \\
\text { esoph. } \\
\text { press. } \\
\text { grad. }\end{array}$} & \multirow{2}{*}{$\begin{array}{l}\text { Inf. } \\
\text { esoph. } \\
\text { sph. } \\
\text { Mean } \\
\text { rest. } \\
\text { end-exp. } \\
\text { press. }\end{array}$} & \multicolumn{2}{|c|}{$\begin{array}{l}\text { Peristaltic press. } \\
\text { Middle } \frac{1}{3} \text { esoph. }\end{array}$} & \multicolumn{2}{|c|}{ Nonprop. cont. } \\
\hline & & & & & Max. & Mean & $\begin{array}{l}\text { \% Total } \\
\text { swallows }\end{array}$ & $\begin{array}{l}\text { Not prec. by } \\
\text { swallow }\end{array}$ \\
\hline & yrs & & \multicolumn{2}{|c|}{$m m \mathrm{Hg}$} & \multicolumn{2}{|c|}{$m m \mathrm{Hg}$} & & \\
\hline P.C. & 23 & $\begin{array}{l}1 \\
2 \\
3 \\
4 \\
5 \\
6\end{array}$ & $\begin{array}{l}4 \\
4 \\
5 \\
4 \\
4 \\
5\end{array}$ & $\begin{array}{l}16 \\
15 \\
12 \\
15 \\
15 \\
12\end{array}$ & $\begin{array}{l}55 \\
45 \\
45 \\
40 \\
50 \\
50\end{array}$ & $\begin{array}{l}40 \\
35 \\
25 \\
25 \\
25 \\
30\end{array}$ & $\begin{array}{r}<5 \\
<5 \\
0 \\
0 \\
<5 \\
0\end{array}$ & $\begin{array}{l}0 \\
\text { Few } \\
0 \\
\text { Few } \\
\text { Few } \\
0\end{array}$ \\
\hline A.M. & 24 & $\begin{array}{l}1 \\
2 \\
3 \\
4 \\
5 \\
6\end{array}$ & $\begin{array}{l}5 \\
6 \\
5 \\
5 \\
5 \\
5\end{array}$ & $\begin{array}{l}12 \\
10 \\
10 \\
14 \\
10 \\
10\end{array}$ & $\begin{array}{l}50 \\
45 \\
40 \\
50 \\
40 \\
45\end{array}$ & $\begin{array}{l}35 \\
35 \\
20 \\
35 \\
25 \\
30\end{array}$ & $\begin{array}{r}25 \\
33 \\
20 \\
0 \\
0 \\
20\end{array}$ & $\begin{array}{l}0 \\
0 \\
0 \\
0 \\
0 \\
0\end{array}$ \\
\hline M.H. & 22 & $\begin{array}{l}1 \\
2 \\
3 \\
4 \\
5 \\
6\end{array}$ & $\begin{array}{l}3 \\
2 \\
3 \\
2 \\
3 \\
2\end{array}$ & $\begin{array}{r}9 \\
8 \\
9 \\
10 \\
8 \\
10\end{array}$ & $\begin{array}{l}45 \\
45 \\
40 \\
45 \\
45 \\
40\end{array}$ & $\begin{array}{l}35 \\
30 \\
30 \\
30 \\
25 \\
25\end{array}$ & $\begin{array}{r}0 \\
<5 \\
0 \\
<5 \\
10 \\
0\end{array}$ & $\begin{array}{l}0 \\
0 \\
0 \\
0 \\
0 \\
0\end{array}$ \\
\hline L.H. & 34 & $\begin{array}{l}1 \\
2 \\
3 \\
4 \\
5 \\
6\end{array}$ & $\begin{array}{l}2 \\
2 \\
2 \\
2 \\
3 \\
2\end{array}$ & $\begin{array}{l}4 \\
6 \\
5 \\
5 \\
5 \\
6\end{array}$ & $\begin{array}{l}90 \\
70 \\
60 \\
60 \\
50 \\
55\end{array}$ & $\begin{array}{l}50 \\
30 \\
45 \\
30 \\
35 \\
40\end{array}$ & $\begin{array}{r}<5 \\
<5 \\
0 \\
0 \\
0 \\
0\end{array}$ & $\begin{array}{l}0 \\
0 \\
0 \\
0 \\
0 \\
0\end{array}$ \\
\hline D.G. & 44 & $\begin{array}{l}1 \\
2 \\
3 \\
4 \\
5 \\
6\end{array}$ & $\begin{array}{l}3 \\
3 \\
3 \\
4 \\
4 \\
4\end{array}$ & $\begin{array}{l}14 \\
12 \\
12 \\
13 \\
10 \\
12\end{array}$ & $\begin{array}{l}60 \\
50 \\
50 \\
50 \\
45 \\
50\end{array}$ & $\begin{array}{l}40 \\
35 \\
35 \\
40 \\
30 \\
25\end{array}$ & $\begin{array}{r}33 \\
10 \\
50 \\
33 \\
0 \\
20\end{array}$ & $\begin{array}{l}\text { Many } \\
\text { Few } \\
\text { Few } \\
\text { Many } \\
\text { Few } \\
\text { Few }\end{array}$ \\
\hline E.L. & 23 & $\begin{array}{l}1 \\
2 \\
3 \\
4 \\
5 \\
6\end{array}$ & $\begin{array}{l}4 \\
4 \\
5 \\
4 \\
4 \\
5\end{array}$ & $\begin{array}{r}9 \\
10 \\
14 \\
9 \\
12 \\
10\end{array}$ & $\begin{array}{l}45 \\
35 \\
45 \\
35 \\
35 \\
35\end{array}$ & $\begin{array}{l}30 \\
25 \\
25 \\
25 \\
20 \\
20\end{array}$ & $\begin{array}{r}5 \\
5 \\
<5 \\
0 \\
0 \\
5\end{array}$ & $\begin{array}{l}0 \\
0 \\
0 \\
0 \\
0 \\
0\end{array}$ \\
\hline H.N. & 22 & $\begin{array}{l}1 \\
2 \\
5 \\
4 \\
5 \\
6\end{array}$ & $\begin{array}{l}4 \\
3 \\
3 \\
3 \\
4 \\
3\end{array}$ & $\begin{array}{r}10 \\
9 \\
9 \\
11 \\
9 \\
10\end{array}$ & $\begin{array}{l}55 \\
50 \\
60 \\
70 \\
65 \\
50\end{array}$ & $\begin{array}{l}40 \\
40 \\
45 \\
40 \\
45 \\
35\end{array}$ & $\begin{array}{r}0 \\
<5 \\
0 \\
<5 \\
0 \\
0\end{array}$ & $\begin{array}{l}0 \\
0 \\
0 \\
0 \\
0 \\
0\end{array}$ \\
\hline A.M. & 27 & $\begin{array}{l}1 \\
2 \\
3 \\
4 \\
5 \\
6\end{array}$ & $\begin{array}{l}6 \\
7 \\
6 \\
7 \\
7 \\
7\end{array}$ & $\begin{array}{l}5 \\
8 \\
5 \\
8 \\
7 \\
8\end{array}$ & $\begin{array}{l}50 \\
50 \\
55 \\
55 \\
45 \\
45\end{array}$ & $\begin{array}{l}35 \\
35 \\
35 \\
35 \\
30 \\
25\end{array}$ & $\begin{array}{r}40 \\
67 \\
67 \\
33 \\
40 \\
5\end{array}$ & $\begin{array}{l}0 \\
\text { Few } \\
\text { Many } \\
\text { Few } \\
\text { Few } \\
0\end{array}$ \\
\hline F.M. & 27 & $\begin{array}{l}1 \\
2 \\
3 \\
4 \\
5 \\
6\end{array}$ & $\begin{array}{l}2 \\
2 \\
2 \\
3 \\
3 \\
2\end{array}$ & $\begin{array}{l}1 \\
3 \\
2 \\
1 \\
2 \\
2\end{array}$ & $\begin{array}{l}45 \\
45 \\
45 \\
45 \\
45 \\
40\end{array}$ & $\begin{array}{l}25 \\
20 \\
25 \\
30 \\
30 \\
20\end{array}$ & $\begin{array}{r}0 \\
0 \\
60 \\
0 \\
40 \\
0\end{array}$ & $\begin{array}{l}0 \\
0 \\
0 \\
0 \\
0 \\
0\end{array}$ \\
\hline
\end{tabular}

* No diffuse spasm or secondary peristalsis occurred in any of the control subjects. 


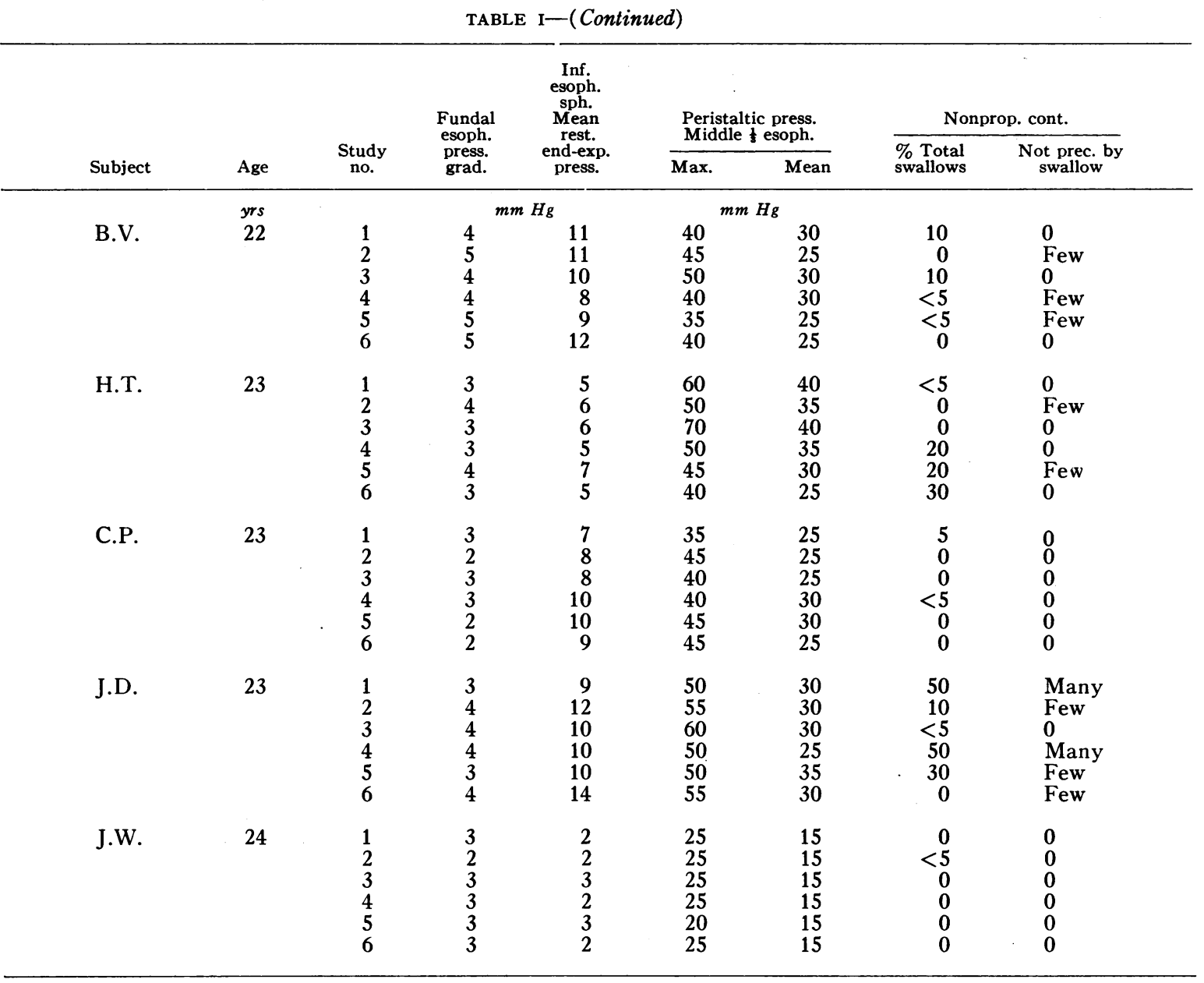

per recording) or "many" (greater than 10 per recording). Each recording extended for approximately 20 minutes. The term "diffuse spasm" (Figure $2 \mathrm{E}$ ) specifically describes forceful, prolonged, repetitive, nonpropulsive contractions in the lower two-thirds of the esophagus (14).

\section{RESULTS}

Control subjects. Although the pressure profile varies among individuals (Table I), certain findings are characteristic of the control group. Diffuse esophageal spasm and secondary peristalsis were not seen. The average fundal-esophageal pressure gradient of 3 to $4 \mathrm{~mm}$ of mercury was constant on serial studies on each subject. The inferior esophageal sphincter (represented by the difference of pressure between the high pressure zone and the gastric fundus) measured from 1 to $16 \mathrm{~mm}$ of mercury. In contrast to the observed variation from one individual to another, serial studies of the sphincter on the same subject generally demonstrated consistently reproducible pressure profiles. The consistency of this pressure response was notable. After a "dry" swallow, a fall in pressure followed by a rise above resting pressure took place in the sphincter region of all control subjects.

Primary peristaltic pressures from 15 to $90 \mathrm{~mm}$ of mercury delineated the normal range. On serial study, 6 subjects inconsistently developed frequent nonpropulsive responses to "dry" swallowing, although a normal peristaltic response invariably followed a swallow of $10 \mathrm{ml}$ of water. Numerous simultaneous contractions not preceded by a swallow occurred in only 3 patients.

Pregnant women without heartburn. As in the control subjects, variability exists among individuals (Table II). Since these women are subject to the physiological effects of an advancing 
TABLE II

Pregnant women without heartburn *

\begin{tabular}{|c|c|c|c|c|c|c|c|c|}
\hline \multirow[b]{2}{*}{ Patient } & \multirow[b]{2}{*}{ Age } & \multirow{2}{*}{$\begin{array}{l}\text { Month of } \\
\text { pregnancy } \\
\text { studied }\end{array}$} & \multirow{2}{*}{$\begin{array}{l}\text { Fundal } \\
\text { esoph. } \\
\text { press. } \\
\text { grad. }\end{array}$} & \multirow{2}{*}{$\begin{array}{l}\text { Inf. } \\
\text { esoph. } \\
\text { sph. } \\
\text { Mean } \\
\text { rest. } \\
\text { end-exp. } \\
\text { press. }\end{array}$} & \multicolumn{2}{|c|}{$\begin{array}{l}\text { Peristaltic press. } \\
\text { Middle } \frac{1}{3} \text { esoph. }\end{array}$} & \multicolumn{2}{|c|}{ Nonprop. cont. } \\
\hline & & & & & Max. & Mean & $\begin{array}{l}\% \text { Total } \\
\text { swallows }\end{array}$ & $\begin{array}{l}\text { Not prec. by } \\
\text { swallow }\end{array}$ \\
\hline D.R. & $\begin{array}{r}y r s \\
32 \mathrm{P}\end{array}$ & $\begin{array}{l}6 \\
8 \\
\text { PP }\end{array}$ & $\begin{array}{ll} & n \\
2 & \\
3 & \\
3 & \end{array}$ & $\begin{array}{r}H g \\
10 \\
6 \\
10\end{array}$ & $\begin{array}{r}80 \\
100 \\
70\end{array}$ & $\begin{array}{l}40 \\
45 \\
35\end{array}$ & $\begin{array}{r}40 \\
50 \\
5\end{array}$ & $\begin{array}{l}0 \\
\text { Few } \\
0\end{array}$ \\
\hline S.M. & $24 \mathrm{P}$ & $\begin{array}{l}4 \\
5 \\
6 \\
7 \\
8 \\
\text { PP }\end{array}$ & $\begin{array}{l}5 \\
6 \\
7 \\
8 \\
9 \\
3\end{array}$ & $\begin{array}{l}10 \\
12 \\
12 \\
10 \\
10 \\
10\end{array}$ & $\begin{array}{l}55 \\
50 \\
50 \\
55 \\
45 \\
50\end{array}$ & $\begin{array}{l}45 \\
40 \\
40 \\
45 \\
35 \\
40\end{array}$ & $\begin{array}{l}25 \\
20 \\
50 \\
40 \\
30 \\
<5\end{array}$ & $\begin{array}{l}0 \\
0 \\
\text { Many } \\
\text { Few } \\
\text { Few } \\
0\end{array}$ \\
\hline P.S. & $26 \mathrm{P}$ & $\begin{array}{l}5 \\
9 \\
\mathrm{PP}\end{array}$ & $\begin{array}{l}2 \\
6 \\
3\end{array}$ & $\begin{array}{r}9 \\
10 \\
8\end{array}$ & $\begin{array}{l}50 \\
40 \\
50\end{array}$ & $\begin{array}{l}30 \\
20 \\
30\end{array}$ & $\begin{array}{r}20 \\
50 \\
0\end{array}$ & $\begin{array}{l}0 \\
0 \\
0\end{array}$ \\
\hline S.K. & $20 \mathrm{M}$ & $\begin{array}{l}7 \\
8 \\
\mathrm{PP}\end{array}$ & $\begin{array}{l}2 \\
9 \\
4\end{array}$ & $\begin{array}{r}10 \\
10 \\
9\end{array}$ & $\begin{array}{l}50 \\
40 \\
45\end{array}$ & $\begin{array}{l}25 \\
25 \\
25\end{array}$ & $\begin{array}{l}30 \\
30 \\
50\end{array}$ & $\begin{array}{l}0 \\
0 \\
0\end{array}$ \\
\hline E.W. & $23 \mathrm{M}$ & $\begin{array}{l}4 \\
5 \\
6 \\
7 \\
8 \\
\text { PP }\end{array}$ & $\begin{array}{l}6 \\
8 \\
8 \\
7 \\
8 \\
5\end{array}$ & $\begin{array}{l}7 \\
6 \\
7 \\
8 \\
7 \\
7\end{array}$ & $\begin{array}{l}60 \\
50 \\
70 \\
55 \\
50 \\
50\end{array}$ & $\begin{array}{l}30 \\
30 \\
40 \\
30 \\
30 \\
30\end{array}$ & $\begin{array}{l}50 \\
70 \\
40 \\
70 \\
70 \\
50\end{array}$ & $\begin{array}{l}0 \\
\text { Many } \\
\text { Few } \\
\text { Few } \\
\text { Many } \\
\text { Many }\end{array}$ \\
\hline J.J. & $28 \mathrm{M}$ & $\begin{array}{l}5 \\
8 \\
\mathrm{PP}\end{array}$ & $\begin{array}{r}8 \\
10 \\
6\end{array}$ & $\begin{array}{r}9 \\
10 \\
12\end{array}$ & $\begin{array}{l}55 \\
50 \\
50\end{array}$ & $\begin{array}{l}30 \\
35 \\
35\end{array}$ & $\begin{array}{l}5 \\
0 \\
0\end{array}$ & $\begin{array}{l}0 \\
0 \\
0\end{array}$ \\
\hline S.F. & $23 \mathrm{P}$ & $\begin{array}{l}4 \\
5 \\
6 \\
7 \\
9 \\
\text { PP }\end{array}$ & $\begin{array}{l}5 \\
4 \\
4 \\
4 \\
5 \\
4\end{array}$ & $\begin{array}{l}6 \\
4 \\
5 \\
6 \\
6 \\
5\end{array}$ & $\begin{array}{l}40 \\
45 \\
30 \\
30 \\
40 \\
35\end{array}$ & $\begin{array}{l}30 \\
20 \\
20 \\
20 \\
25 \\
20\end{array}$ & $\begin{array}{r}50 \\
70 \\
30 \\
66 \\
50 \\
<5\end{array}$ & $\begin{array}{l}0 \\
\text { Many } \\
\text { Few } \\
\text { Many } \\
\text { Many } \\
\text { Few }\end{array}$ \\
\hline S.B. & $21 \mathrm{P}$ & $\begin{array}{l}6 \\
7 \\
9 \\
\text { PP }\end{array}$ & $\begin{array}{l}8 \\
9 \\
8 \\
6\end{array}$ & $\begin{array}{l}12 \\
10 \\
10 \\
10\end{array}$ & $\begin{array}{l}50 \\
55 \\
50 \\
50\end{array}$ & $\begin{array}{l}30 \\
40 \\
30 \\
35\end{array}$ & $\begin{array}{r}80 \\
40 \\
33 \\
0\end{array}$ & $\begin{array}{l}\text { Many } \\
\text { Few } \\
\mathbf{0} \\
\mathbf{0}\end{array}$ \\
\hline N.S. & $22 \mathrm{M}$ & $\begin{array}{l}8 \\
\text { PP }\end{array}$ & $\begin{array}{l}4 \\
3\end{array}$ & $\begin{array}{l}2 \\
3\end{array}$ & $\begin{array}{l}25 \\
35\end{array}$ & $\begin{array}{l}15 \\
20\end{array}$ & $\begin{array}{l}90 \\
60\end{array}$ & $\begin{array}{l}\text { Few } \\
\text { Few }\end{array}$ \\
\hline C.S. & $26 \mathrm{P}$ & $\begin{array}{l}7 \\
\text { PP }\end{array}$ & $\begin{array}{l}5 \\
5\end{array}$ & $\begin{array}{r}6 \\
10\end{array}$ & $\begin{array}{l}50 \\
55\end{array}$ & $\begin{array}{l}35 \\
35\end{array}$ & $\begin{array}{l}70 \\
25\end{array}$ & $\begin{array}{l}\text { Few } \\
\text { Many }\end{array}$ \\
\hline A.E. & $26 \mathrm{M}$ & $\begin{array}{l}8 \\
P P\end{array}$ & $\begin{array}{l}5 \\
2\end{array}$ & $\begin{array}{l}5 \\
5\end{array}$ & $\begin{array}{l}50 \\
50\end{array}$ & $\begin{array}{l}40 \\
30\end{array}$ & $\begin{array}{r}30 \\
0\end{array}$ & $\begin{array}{l}\text { Few } \\
\text { Few }\end{array}$ \\
\hline E.V. & $35 \mathrm{M}$ & $\begin{array}{l}6 \\
8 \\
\text { PP }\end{array}$ & $\begin{array}{l}3 \\
7 \\
2\end{array}$ & $\begin{array}{r}7 \\
3 \\
10\end{array}$ & $\begin{array}{l}25 \\
20 \\
25\end{array}$ & $\begin{array}{l}15 \\
15 \\
15\end{array}$ & $\begin{array}{r}70 \\
30 \\
<5\end{array}$ & $\begin{array}{l}\text { Few } \\
\mathbf{0} \\
\mathbf{0}\end{array}$ \\
\hline M.S. & $27 \mathrm{M}$ & $\begin{array}{l}8 \\
9 \\
\text { PP }\end{array}$ & $\begin{array}{l}5 \\
5 \\
2\end{array}$ & $\begin{array}{l}1 \\
2 \\
5\end{array}$ & $\begin{array}{l}45 \\
40 \\
40\end{array}$ & $\begin{array}{l}25 \\
20 \\
20\end{array}$ & $\begin{array}{r}40 \\
50 \\
<5\end{array}$ & $\begin{array}{l}0 \\
\text { Few } \\
0\end{array}$ \\
\hline I.G. & $35 \mathrm{M}$ & $\stackrel{8}{\mathrm{PP}}$ & $\begin{array}{l}4 \\
4\end{array}$ & $\begin{array}{l}8 \\
9\end{array}$ & $\begin{array}{l}45 \\
50\end{array}$ & $\begin{array}{l}35 \\
30\end{array}$ & $\begin{array}{r}10 \\
0\end{array}$ & $\begin{array}{l}0 \\
0\end{array}$ \\
\hline
\end{tabular}

${ }^{*} \mathrm{P}=$ primipara $; \mathrm{M}=$ multipara $; \mathrm{PP}=$ post partum. No diffuse spasm or secondary peristalsis occurred in the pregnant women without heartburn. 
TABLE II-(Continued)

\begin{tabular}{|c|c|c|c|c|c|c|c|c|}
\hline \multirow[b]{2}{*}{ Patient } & \multirow[b]{2}{*}{ Age } & \multirow{2}{*}{$\begin{array}{l}\text { Month of } \\
\text { pregnancy } \\
\text { studied }\end{array}$} & \multirow{2}{*}{$\begin{array}{l}\text { Fundal } \\
\text { esoph. } \\
\text { press. } \\
\text { grad. }\end{array}$} & \multirow{2}{*}{$\begin{array}{l}\text { Inf. } \\
\text { esoph. } \\
\text { sph. } \\
\text { Mean } \\
\text { rest. } \\
\text { end-exp. } \\
\text { press. }\end{array}$} & \multicolumn{2}{|c|}{$\begin{array}{l}\text { Peristaltic press. } \\
\text { Middle } \frac{1}{3} \text { esoph. }\end{array}$} & \multicolumn{2}{|c|}{ Nonprop. cont. } \\
\hline & & & & & Max. & Mean & $\begin{array}{l}\text { \% Total } \\
\text { swallows }\end{array}$ & $\begin{array}{l}\text { Not prec. by } \\
\text { swallow }\end{array}$ \\
\hline L.W. & $\begin{array}{r}y r s \\
26 \mathrm{P}\end{array}$ & $\begin{array}{l}6 \\
8 \\
9 \\
\mathrm{PP}\end{array}$ & $\begin{array}{l}2 \\
2 \\
4 \\
6 \\
2\end{array}$ & $\begin{array}{l}5 \\
3 \\
2 \\
6\end{array}$ & $\begin{array}{l}30 \\
35 \\
35 \\
35\end{array}$ & $\begin{array}{l}15 \\
20 \\
15 \\
15\end{array}$ & $\begin{array}{r}60 \\
25 \\
30 \\
<5\end{array}$ & $\begin{array}{l}\text { Few } \\
\text { Few } \\
0 \\
0\end{array}$ \\
\hline D.G. & $23 \mathrm{P}$ & $\begin{array}{l}6 \\
8 \\
\text { PP }\end{array}$ & $\begin{array}{l}5 \\
8 \\
3\end{array}$ & $\begin{array}{l}5 \\
6 \\
5\end{array}$ & $\begin{array}{l}45 \\
50 \\
40\end{array}$ & $\begin{array}{l}30 \\
30 \\
25\end{array}$ & $\begin{array}{l}80 \\
60 \\
60\end{array}$ & $\begin{array}{l}\text { Few } \\
\text { Few } \\
\text { Few }\end{array}$ \\
\hline F.D. & $25 \mathrm{P}$ & $\stackrel{8}{\mathrm{PP}}$ & $\begin{array}{l}5 \\
3\end{array}$ & $\begin{array}{l}6 \\
5\end{array}$ & $\begin{array}{l}35 \\
35\end{array}$ & $\begin{array}{l}10 \\
25\end{array}$ & $\begin{array}{r}60 \\
0\end{array}$ & $\begin{array}{l}0 \\
0\end{array}$ \\
\hline N.H. & $24 \mathrm{M}$ & $\stackrel{9}{\mathrm{PP}}$ & $\begin{array}{l}7 \\
3\end{array}$ & $\begin{array}{l}6 \\
7\end{array}$ & $\begin{array}{l}40 \\
45\end{array}$ & $\begin{array}{l}25 \\
20\end{array}$ & $\begin{array}{l}40 \\
60\end{array}$ & $\begin{array}{l}\text { Many } \\
\text { Few }\end{array}$ \\
\hline C.C. & $31 \mathrm{P}$ & $\begin{array}{l}6 \\
8 \\
\text { PP }\end{array}$ & $\begin{array}{l}5 \\
8 \\
5\end{array}$ & $\begin{array}{l}10 \\
12 \\
10\end{array}$ & $\begin{array}{l}55 \\
65 \\
80\end{array}$ & $\begin{array}{l}35 \\
40 \\
40\end{array}$ & $\begin{array}{l}50 \\
30 \\
40\end{array}$ & $\begin{array}{l}\text { O } \\
0 \\
\text { Few }\end{array}$ \\
\hline
\end{tabular}

pregnancy, the results of serial esophageal motility changes during pregnancy are presented, each woman serving as her own control. Neither diffuse esophageal spasm nor secondary peristalsis was seen. The average fundal esophageal pressure gradient of 6 to $7 \mathrm{~mm}$ of mercury was higher than that recorded in the controls. This gradient generally rose with advancing pregnancy and fell post partum to levels similar to those determined in the normal nonpregnant group. The mean resting end-expiratory pressure developed by the inferior esophageal sphincter varied from 1 to $12 \mathrm{~mm}$ of mercury. Fourteen women maintained a consistent sphincter pressure profile throughout pregnancy and after delivery, while five subjects demonstrated a fall in sphincteric pressure as pregnancy advanced, with a return to previous levels post partum. After a "dry" swallow, a fall in sphincter pressure followed by a rise above resting levels occurred in all but two patients during pregnancy. Both of these women demonstrated a barely perceptible high pressure zone. The return of normal sphincteric function after delivery coincided with a rise in the resting sphincter pressure. During pregnancy, the inferior esophageal sphincter was confined at or just above the effective diaphragmatic hiatus in 63 per cent of subjects. This represented a significant departure from its usual anatomical position, which in most women ordinarily included an intra-abdominal segment.

Primary peristaltic pressures ranged from 10 to $100 \mathrm{~mm}$ of mercury and compared closely with control values. Seventeen women consistently developed frequent nonpropulsive pressure responses to "dry" swallowing during pregnancy, although a normal peristaltic wave usually followed a swallow of $10 \mathrm{ml}$ of water. Six women showed numerous nonpropulsive contractions not preceded by a swallow. After delivery, the incidence of abnormal motor responses approached the controls. Neither parity nor age correlated with the changes observed.

Pregnant women with heartburn. The results (Table III) illustrate the changing esophageal pressure profile during pregnancy, each woman serving as her own control. Forty-five per cent demonstrated secondary peristalsis which disappeared post partum, but which was never seen in either of the other two groups. The average fundal esophageal pressure gradient of 6 to $7 \mathrm{~mm}$ of mercury was similar to that of the group of pregnant women without heartburn, but higher than that of the controls. This gradient generally rose with advancing pregnancy and fell post partum to levels similar to those found in the normal nonpregnant group. The mean resting end-expiratory pressure of the inferior esophageal 
TABLE III

Pregnant women with heartburn *

\begin{tabular}{|c|c|c|c|c|c|c|c|c|c|c|}
\hline \multirow[b]{2}{*}{ Patient } & \multirow[b]{2}{*}{ Age } & \multirow{2}{*}{$\begin{array}{l}\text { Month of } \\
\text { pregnancy } \\
\text { studied }\end{array}$} & \multirow{2}{*}{$\begin{array}{l}\text { Fundal } \\
\text { esoph. } \\
\text { press. } \\
\text { grad. }\end{array}$} & \multirow{2}{*}{$\begin{array}{l}\text { Inf. } \\
\text { esoph. } \\
\text { sph. } \\
\text { Mean } \\
\text { rest. } \\
\text { end-exp. } \\
\text { press. }\end{array}$} & \multicolumn{2}{|c|}{$\begin{array}{l}\text { Peristaltic press. } \\
\text { Middle } \frac{1}{3} \text { esoph. }\end{array}$} & \multicolumn{2}{|c|}{ Nonprop. cont. } & \multirow[b]{2}{*}{$\begin{array}{l}\text { Diffuse } \\
\text { spasm }\end{array}$} & \multirow[b]{2}{*}{$\begin{array}{l}\text { Secondary } \\
\text { peristalsis }\end{array}$} \\
\hline & & & & & Max. & Mean & $\begin{array}{l}\text { \% Total } \\
\text { swallows }\end{array}$ & $\begin{array}{l}\text { Not prec. by } \\
\text { swallow }\end{array}$ & & \\
\hline J.W. & $\begin{array}{r}y r s \\
21 \mathrm{P}\end{array}$ & $\begin{array}{l}7 \\
9 \\
\mathrm{PP}\end{array}$ & $\begin{array}{ll} & m \eta \\
3 & \\
5 & \end{array}$ & $\begin{array}{r}\mathrm{Hg} \\
5 \\
3 \\
8\end{array}$ & $\begin{array}{l}{ }^{m} \\
45 \\
45\end{array}$ & $\begin{array}{r}I g \\
50 \\
25 \\
30\end{array}$ & $\begin{array}{r}<5 \\
0 \\
0\end{array}$ & $\begin{array}{l}0 \\
0 \\
0\end{array}$ & & + \\
\hline D.B. & $28 \mathrm{M}$ & $\begin{array}{l}9 \\
\mathrm{PP}\end{array}$ & $\begin{array}{l}8 \\
4\end{array}$ & $\begin{array}{l}1 \\
6\end{array}$ & $\begin{array}{l}55 \\
45\end{array}$ & $\begin{array}{l}40 \\
35\end{array}$ & $\begin{array}{r}<5 \\
0\end{array}$ & $\begin{array}{l}0 \\
0\end{array}$ & & \\
\hline R.T. & $29 \mathrm{M}$ & $\begin{array}{l}9 \\
\text { PP }\end{array}$ & $\begin{array}{l}3 \\
4\end{array}$ & $\begin{array}{l}7 \\
8\end{array}$ & $\begin{array}{r}90 \\
140\end{array}$ & $\begin{array}{l}45 \\
70\end{array}$ & $\begin{array}{l}90 \\
90\end{array}$ & $\begin{array}{l}\text { Many } \\
\text { Few }\end{array}$ & $\begin{array}{l}+ \\
+\end{array}$ & \\
\hline A.D. & $30 \mathrm{P}$ & $\begin{array}{l}6 \\
7 \\
8 \\
\mathrm{PP}\end{array}$ & $\begin{array}{l}5 \\
7 \\
8 \\
3\end{array}$ & $\begin{array}{r}9 \\
6 \\
3 \\
10\end{array}$ & $\begin{array}{l}45 \\
35 \\
45 \\
45\end{array}$ & $\begin{array}{l}35 \\
25 \\
30 \\
25\end{array}$ & $\begin{array}{r}<5 \\
10 \\
80 \\
0\end{array}$ & $\begin{array}{l}0 \\
0 \\
\text { Few } \\
0\end{array}$ & & + \\
\hline R.K. & $31 \mathrm{M}$ & $\begin{array}{l}8 \\
9 \\
\text { PP }\end{array}$ & $\begin{array}{l}5 \\
7 \\
3\end{array}$ & $\begin{array}{l}5 \\
6 \\
6\end{array}$ & $\begin{array}{l}50 \\
40 \\
40\end{array}$ & $\begin{array}{l}25 \\
25 \\
20\end{array}$ & $\begin{array}{r}5 \\
10 \\
<5\end{array}$ & $\begin{array}{l}0 \\
0 \\
0\end{array}$ & & \\
\hline B.H. & $25 \mathrm{P}$ & $\stackrel{8}{\mathrm{PP}}$ & $\begin{array}{l}8 \\
4\end{array}$ & $\begin{array}{l}8 \\
7\end{array}$ & $\begin{array}{l}60 \\
70\end{array}$ & $\begin{array}{l}40 \\
40\end{array}$ & $\begin{array}{l}75 \\
70\end{array}$ & $\begin{array}{l}\text { Few } \\
\text { Many }\end{array}$ & + & \\
\hline B.R. & $25 \mathrm{M}$ & $\begin{array}{l}6 \\
8 \\
\mathrm{PP}\end{array}$ & $\begin{array}{l}5 \\
9 \\
4\end{array}$ & $\begin{array}{r}12 \\
6 \\
10\end{array}$ & $\begin{array}{l}50 \\
50 \\
70\end{array}$ & $\begin{array}{l}35 \\
30 \\
45\end{array}$ & $\begin{array}{l}20 \\
50 \\
30\end{array}$ & $\begin{array}{l}\text { Many } \\
\text { Few } \\
\text { Few }\end{array}$ & & \\
\hline H.U. & $39 M$ & $\begin{array}{l}8 \\
9 \\
\mathrm{PP}\end{array}$ & $\begin{array}{l}6 \\
8 \\
5\end{array}$ & $\begin{array}{l}4 \\
5 \\
5\end{array}$ & $\begin{array}{l}30 \\
30 \\
30\end{array}$ & $\begin{array}{l}15 \\
25 \\
20\end{array}$ & $\begin{array}{r}50 \\
70 \\
5\end{array}$ & $\begin{array}{l}0 \\
0 \\
0\end{array}$ & & \\
\hline J.H. & $29 \mathrm{P}$ & $\begin{array}{l}9 \\
\mathrm{PP}\end{array}$ & $\begin{array}{l}4 \\
3\end{array}$ & $\begin{array}{l}6 \\
5\end{array}$ & $\begin{array}{l}80 \\
95\end{array}$ & $\begin{array}{l}35 \\
40\end{array}$ & $\begin{array}{l}75 \\
50\end{array}$ & $\begin{array}{l}\text { Many } \\
\text { Many }\end{array}$ & + & \\
\hline M.R. & $25 \mathrm{M}$ & $\begin{array}{l}4 \\
6 \\
7 \\
8 \\
9 \\
\mathrm{PP}\end{array}$ & $\begin{array}{l}5 \\
6 \\
8 \\
9 \\
8 \\
5\end{array}$ & $\begin{array}{l}5 \\
4 \\
3 \\
2 \\
2 \\
6\end{array}$ & $\begin{array}{l}20 \\
30 \\
30 \\
25 \\
20 \\
20\end{array}$ & $\begin{array}{l}15 \\
15 \\
10 \\
10 \\
10 \\
10\end{array}$ & $\begin{array}{l}75 \\
60 \\
90 \\
60 \\
90 \\
<5\end{array}$ & $\begin{array}{l}\text { Few } \\
\text { Few } \\
\text { Many } \\
\text { Many } \\
\text { Many } \\
\text { Few }\end{array}$ & & $\begin{array}{l}+ \\
+\end{array}$ \\
\hline R.C. & $35 \mathrm{M}$ & $\begin{array}{l}8 \\
9 \\
\mathrm{PP}\end{array}$ & $\begin{array}{l}7 \\
6 \\
2\end{array}$ & $\begin{array}{l}12 \\
15 \\
12\end{array}$ & $\begin{array}{r}100 \\
110 \\
80\end{array}$ & $\begin{array}{l}50 \\
50 \\
35\end{array}$ & $\begin{array}{l}67 \\
67 \\
33\end{array}$ & $\begin{array}{l}0 \\
0 \\
0\end{array}$ & & \\
\hline P.M. & $21 \mathrm{P}$ & $\begin{array}{l}9 \\
\mathrm{PP}\end{array}$ & $\begin{array}{l}8 \\
3\end{array}$ & $\begin{array}{l}2 \\
6\end{array}$ & $\begin{array}{l}40 \\
50\end{array}$ & $\begin{array}{l}30 \\
40\end{array}$ & $\begin{array}{l}<5 \\
<5\end{array}$ & $\begin{array}{l}0 \\
0\end{array}$ & & + \\
\hline J.L. & $27 \mathrm{M}$ & $\begin{array}{l}8 \\
9 \\
\text { PP }\end{array}$ & $\begin{array}{l}7 \\
7 \\
2\end{array}$ & $\begin{array}{l}2 \\
1 \\
4\end{array}$ & $\begin{array}{l}60 \\
50 \\
50\end{array}$ & $\begin{array}{l}40 \\
35 \\
35\end{array}$ & $\begin{array}{r}50 \\
50 \\
0\end{array}$ & $\begin{array}{l}\text { Few } \\
\text { Few } \\
0\end{array}$ & & $\begin{array}{l}+ \\
+\end{array}$ \\
\hline C.F. & $26 \mathrm{M}$ & $\begin{array}{l}7 \\
8 \\
\mathrm{PP}\end{array}$ & $\begin{array}{l}4 \\
5 \\
5\end{array}$ & $\begin{array}{l}6 \\
5 \\
9\end{array}$ & $\begin{array}{l}45 \\
50 \\
45\end{array}$ & $\begin{array}{l}35 \\
35 \\
30\end{array}$ & $\begin{array}{r}30 \\
50 \\
5\end{array}$ & $\begin{array}{l}\text { Few } \\
\text { Few } \\
0\end{array}$ & & \\
\hline E.H. & $35 \mathrm{M}$ & $\begin{array}{l}9 \\
\text { PP }\end{array}$ & $\begin{array}{l}9 \\
3\end{array}$ & $\begin{array}{l}0 \\
6\end{array}$ & $\begin{array}{l}40 \\
45\end{array}$ & $\begin{array}{l}20 \\
25\end{array}$ & $\begin{array}{l}0 \\
0\end{array}$ & $\begin{array}{l}0 \\
0\end{array}$ & & + \\
\hline D.W. & $23 \mathrm{P}$ & $\begin{array}{l}9 \\
\mathrm{PP}\end{array}$ & $\begin{array}{l}8 \\
5\end{array}$ & $\begin{array}{l}2 \\
3\end{array}$ & $\begin{array}{l}25 \\
40\end{array}$ & $\begin{array}{l}15 \\
20\end{array}$ & $\begin{array}{l}60 \\
50\end{array}$ & $\begin{array}{l}\text { Few } \\
0\end{array}$ & & + \\
\hline
\end{tabular}

$* \mathrm{P}=$ primipara $; \mathrm{M}=$ multipara $; \mathrm{PP}=$ post partum. 


\begin{tabular}{|c|c|c|c|c|c|c|c|c|c|c|}
\hline \multirow[b]{2}{*}{ Patient } & \multirow[b]{2}{*}{ Age } & \multirow{2}{*}{$\begin{array}{l}\text { Month of } \\
\text { pregnancy } \\
\text { studied }\end{array}$} & \multirow{2}{*}{$\begin{array}{l}\text { Fundal } \\
\text { esoph. } \\
\text { press. } \\
\text { grad. }\end{array}$} & \multirow{2}{*}{$\begin{array}{l}\text { Inf. } \\
\text { esoph. } \\
\text { sph. } \\
\text { Mean } \\
\text { rest. } \\
\text { end-exp. } \\
\text { press. }\end{array}$} & \multicolumn{2}{|c|}{$\begin{array}{l}\text { Peristaltic press. } \\
\text { Middle } \frac{1}{3} \text { esoph. }\end{array}$} & \multicolumn{2}{|c|}{ Nonprop. cont. } & \multirow[b]{2}{*}{$\begin{array}{l}\text { Diffuse } \\
\text { spasm }\end{array}$} & \multirow[b]{2}{*}{$\begin{array}{l}\text { Secondary } \\
\text { peristalsis }\end{array}$} \\
\hline & & & & & $\frac{\text { Middl }}{\text { Max. }}$ & $\frac{\text { esoph. }}{\text { Mean }}$ & $\begin{array}{l}\text { \% Total } \\
\text { swallows }\end{array}$ & $\begin{array}{c}\text { Not prec. by } \\
\text { swallow }\end{array}$ & & \\
\hline & $y r s$ & & \multicolumn{2}{|c|}{$\mathrm{mm} \mathrm{Hg}$} & \multicolumn{2}{|c|}{$m m ~ H g$} & & & & \\
\hline H.C. & $38 \mathrm{M}$ & $\begin{array}{l}9 \\
\mathrm{PP}\end{array}$ & $\begin{array}{l}5 \\
2\end{array}$ & $\begin{array}{l}5 \\
5\end{array}$ & $\begin{array}{l}45 \\
40\end{array}$ & $\begin{array}{l}30 \\
25\end{array}$ & $\begin{array}{l}80 \\
20\end{array}$ & $\begin{array}{l}\text { Many } \\
0\end{array}$ & & + \\
\hline E.M. & $16 \mathrm{P}$ & $\begin{array}{l}9 \\
\mathrm{PP}\end{array}$ & $\begin{array}{l}5 \\
4\end{array}$ & $\begin{array}{l}2 \\
6\end{array}$ & $\begin{array}{l}45 \\
55\end{array}$ & $\begin{array}{l}25 \\
30\end{array}$ & $\begin{array}{r}60 \\
0\end{array}$ & $\begin{array}{l}\text { Few } \\
\text { Few }\end{array}$ & & \\
\hline A.V. & $18 \mathrm{P}$ & $\begin{array}{l}7 \\
9 \\
\mathrm{PP}\end{array}$ & $\begin{array}{l}6 \\
7 \\
4\end{array}$ & $\begin{array}{r}8 \\
4 \\
10\end{array}$ & $\begin{array}{l}55 \\
45 \\
45\end{array}$ & $\begin{array}{l}30 \\
20 \\
25\end{array}$ & $\begin{array}{l}50 \\
75 \\
50\end{array}$ & $\begin{array}{l}\text { Few } \\
\text { Few } \\
\text { Few }\end{array}$ & & \\
\hline E.D. & $30 \mathrm{M}$ & $\begin{array}{l}5 \\
6 \\
7 \\
9 \\
\mathrm{PP}\end{array}$ & $\begin{array}{l}3 \\
5 \\
6 \\
8 \\
2\end{array}$ & $\begin{array}{l}6 \\
3 \\
3 \\
2 \\
8\end{array}$ & $\begin{array}{l}40 \\
50 \\
50 \\
45 \\
40\end{array}$ & $\begin{array}{l}25 \\
25 \\
30 \\
20 \\
25\end{array}$ & $\begin{array}{r}50 \\
33 \\
75 \\
50 \\
<5\end{array}$ & $\begin{array}{l}0 \\
\text { Few } \\
\text { Many } \\
\text { Many } \\
\text { Few }\end{array}$ & & + \\
\hline
\end{tabular}

sphincter varied from 0 to $15 \mathrm{~mm}$ of mercury. Eight women maintained a consistent sphincter pressure profile during pregnancy and post partum while 12 demonstrated a fall in sphincteric pres- sure which returned to previous levels after delivery. The response to a "dry" swallow was present in all but two patients during pregnancy. In one woman the inferior esophageal sphincter

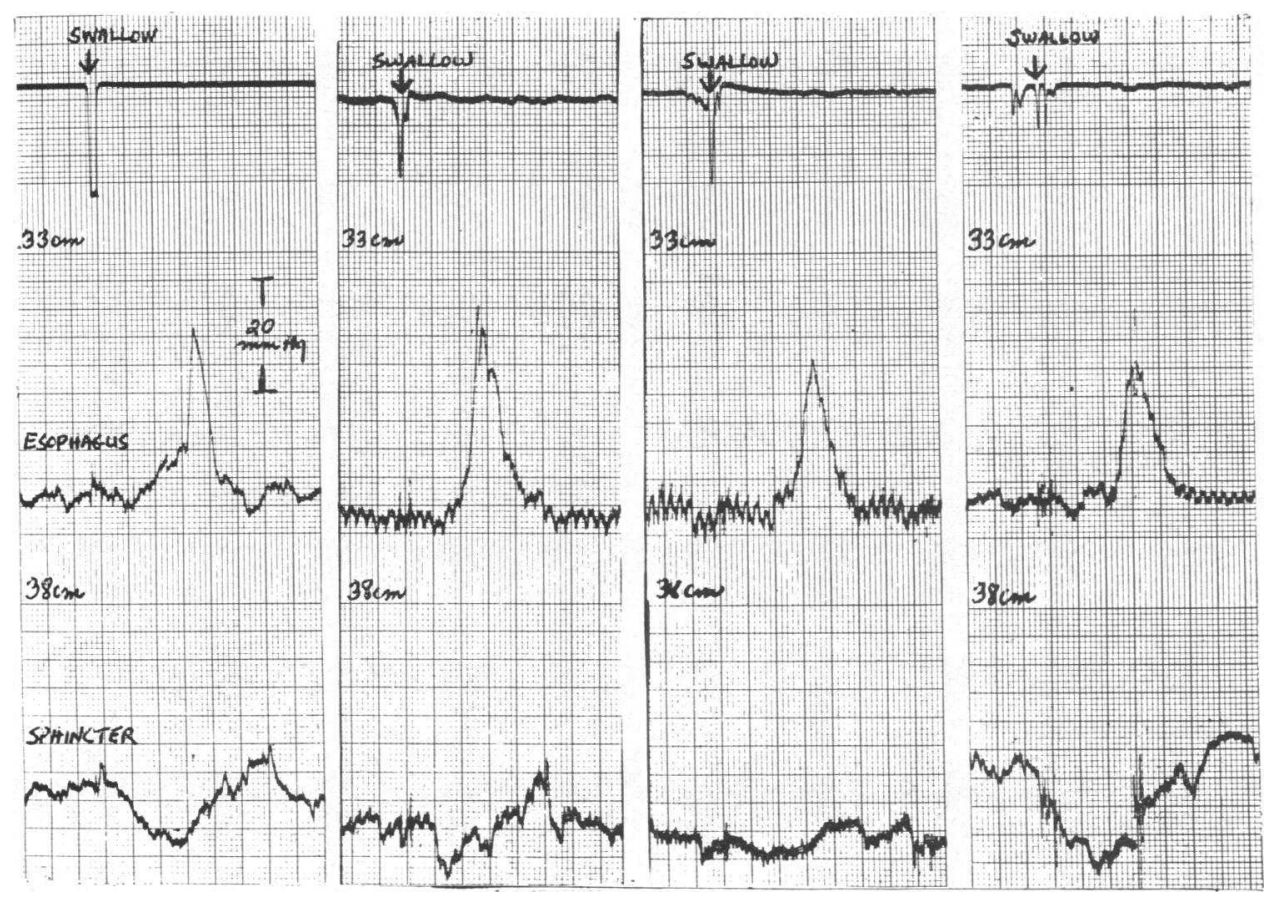

Fig. 3. Electromanometric recordings of Patient E. D. in the fifth, Seventh and EIGHTH MONTHS OF PREgNANCY, AND AFTER Delivery (LEFT to RIGHT). Note the progressive diminution of inferior esophageal sphincter response to a "dry" swallow with advancing pregnancy and the return to normal post partum. The patient experienced heartburn frequently in the last trimester of pregnancy. Calibrations are the same throughout. The numbers on the left of each panel are distances from the incisors. 

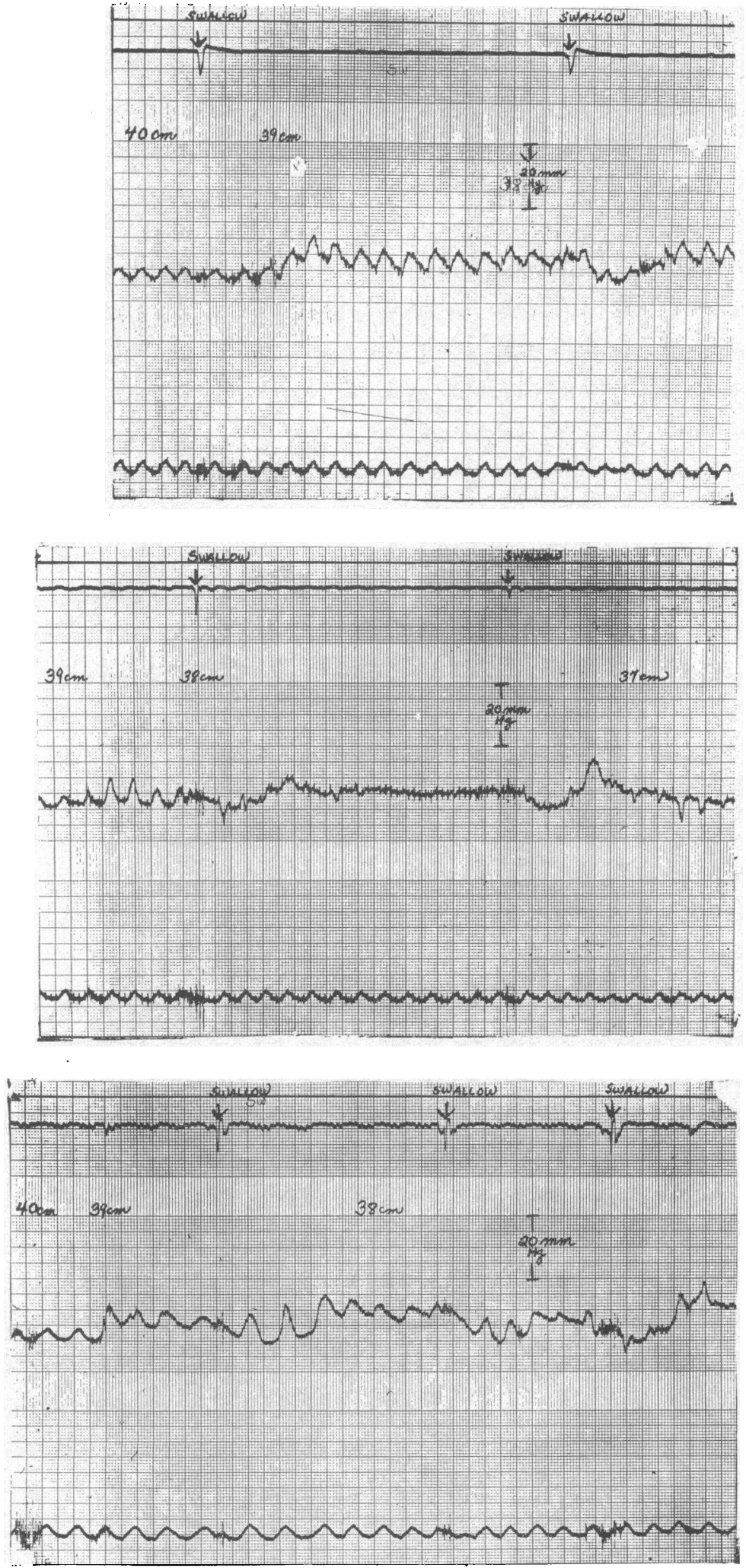

Fig. 4. Electromanometric ReCORDINGS OF PATIENT M. R. IN THE FOURTH (TOP PANEL) AND SEVENTH (MIDDLE PANEL) MONTHS OF PREGNANCY, AND AFTER DELIVERY (BOTTOM PANEL). The numbers across the top of each panel are distances from the incisors. The middle tracing on each panel is from the sphincter; the bottom tracing is from the fundus. Top panel: Respiratory responses in the fundus and sphincter are the same indicating that the sphincter is below the effective diaphragmatic hiatus, i.e., an intra-abdominal segment first identified $39 \mathrm{~cm}$ from the incisors. Middle panel: Respiratory responses in the fundus and sphincter are opposite indicating that the entire sphincter is in the thorax beginning $38 \mathrm{~cm}$ from the incisors at the point of respiratory reversal. Bottom panel: The intraabdominal segment of the sphincter reappears $39 \mathrm{~cm}$ from the incisors. Several drops in pressure following a swallow are evident. 

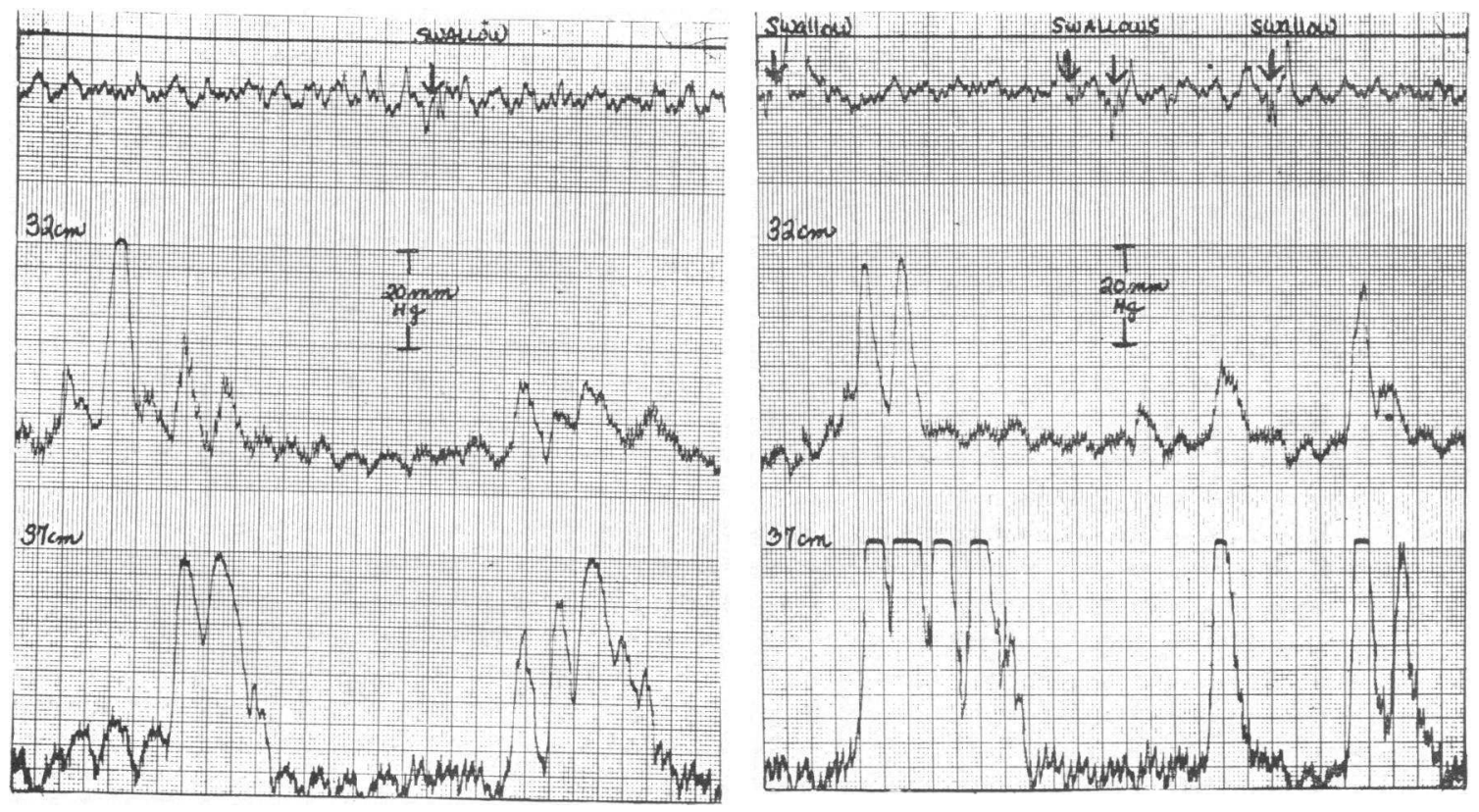

Fig. 5. Electromanometric recordings of Patient B. H. in the eighth month of pregnancy (left) and 2 MONTHS AFTER DELIVERY (RIGHT). The numbers at the left of each panel are distances from the incisors. Left panel: Diffuse esophageal spasm. The patient had severe heartburn during the third trimester of pregnancy. Right panel: Diffuse esophageal spasm still present. The patient had been completely asymptomatic since delivery.

could not be demonstrated, and in the other it was barely perceptible (Figure 3). Both patients, however, revealed normal function post partum, coincident with a substantial rise in the resting sphincteric pressure. A most important finding during pregnancy was that an intra-abdominal segment of the sphincter was identified in only 15 per cent of women in this group; it reappeared after delivery in most subjects (Figure 4).

Three women had a pressure profile typical of diffuse esophageal spasm, following both a "dry" and "wet" swallow, but in the asymptomatic postpartum period, motility remained similarly disturbed (Figure 5). In each instance, the inferior esophageal sphincter was well developed and demonstrated normal function.

Primary peristaltic pressures varied from 10 to $110 \mathrm{~mm}$ of mercury, which compared elosely with control values. Fifteen women consistently developed frequent nonpropulsive pressure responses to "dry" swallowing during pregnancy, although a normal primary peristaltic wave usually followed a swallow of $10 \mathrm{ml}$ of water. Seven women showed numerous simultaneous contractions not preceded by a swallow. After delivery, the frequency of abnormal motor responses approached control values. Neither parity nor age correlated with these results.

Three women experienced heartburn during the manometric study and are reported in greater detail.

Subject 1. J. L., a 27 year old gravida II, had heartburn for the first time in the sixth month of pregnancy. Episodes of a burning, hot sensation localized to the throat, associated with the occurrence of sour-tasting fluid in the mouth, were brought on by large meals and the supine position. Antacids gave prompt relief. During an esophageal motility study performed in the eighth month of pregnancy, the patient complained of waves of heartburn; simultaneously, secondary peristaltic waves appeared on the graph (Figure 6). The fundal esophageal pressure gradient was $7 \mathrm{~mm}$ of mercury. The inferior esophageal sphincter was located entirely above the effective diaphragmatic hiatus and developed a mean resting end-expiratory pressure of $2 \mathrm{~mm}$ of mercury. Numerous nonpropulsive pressures developed both spontaneously and in response to swallowing. During the ninth month of pregnancy, heartburn again was associated with the appearance of secondary peristaltic waves. With the catheters in place, the pa- 
tient was given $30 \mathrm{ml}$ of a liquid antacid which promptly relieved the heartburn. The motility record, however, was unchanged.

After delivery the patient became asymptomatic and a recording 6 weeks later was normal. The fundal esophageal pressure gradient fell to $2 \mathrm{~mm}$ of mercury and the inferior esophageal sphincter which was now observed, both below and above the effective diaphragmatic hiatus, developed a resting pressure of $4 \mathrm{~mm}$ of mercury.

Comment. Esophageal motility studies demonstrated secondary peristalsis simultaneously with symptoms of heartburn. Nonpropulsive activity was also present. A liquid antacid effectively relieved the heartburn, but the pressure profile was unchanged, suggesting that motor phenomena are not the only determinants of heartburn.

Subject 2. E. H., a 35 year old gravida II, had heartburn for the first time in the seventh month of pregnancy. A burning sensation in the substernal area radiating to the throat was precipitated by bending, stooping, or lying flat especially after eating. All antacid preparations nauseated her. The patient complained of persistent heartburn during an esophageal motility study performed in the ninth month of pregnancy, and again secondary peristalsis was seen (Figure 7). The fundal esophageal pressure gradient was $9 \mathrm{~mm}$ of mercury, the inferior esophageal sphincter could not be identified, and nonpropulsive activity was not observed.

The pressure profile was normal 8 weeks post partum; she had experienced no heartburn after delivery. The fundal esophageal pressure gradient fell to $3 \mathrm{~mm}$ of mercury and the inferior esophageal sphincter could now be identified both below and above the effective diaphragmatic hiatus with a mean resting end-expiratory pressure of 6 $\mathrm{mm}$ of mercury. A pneumatic bag was then placed around the patient's waist and $300 \mathrm{ml}$ of water rapidly instilled directly into the stomach. Upon inflation of the bag to exert a pressure of $100 \mathrm{~mm}$

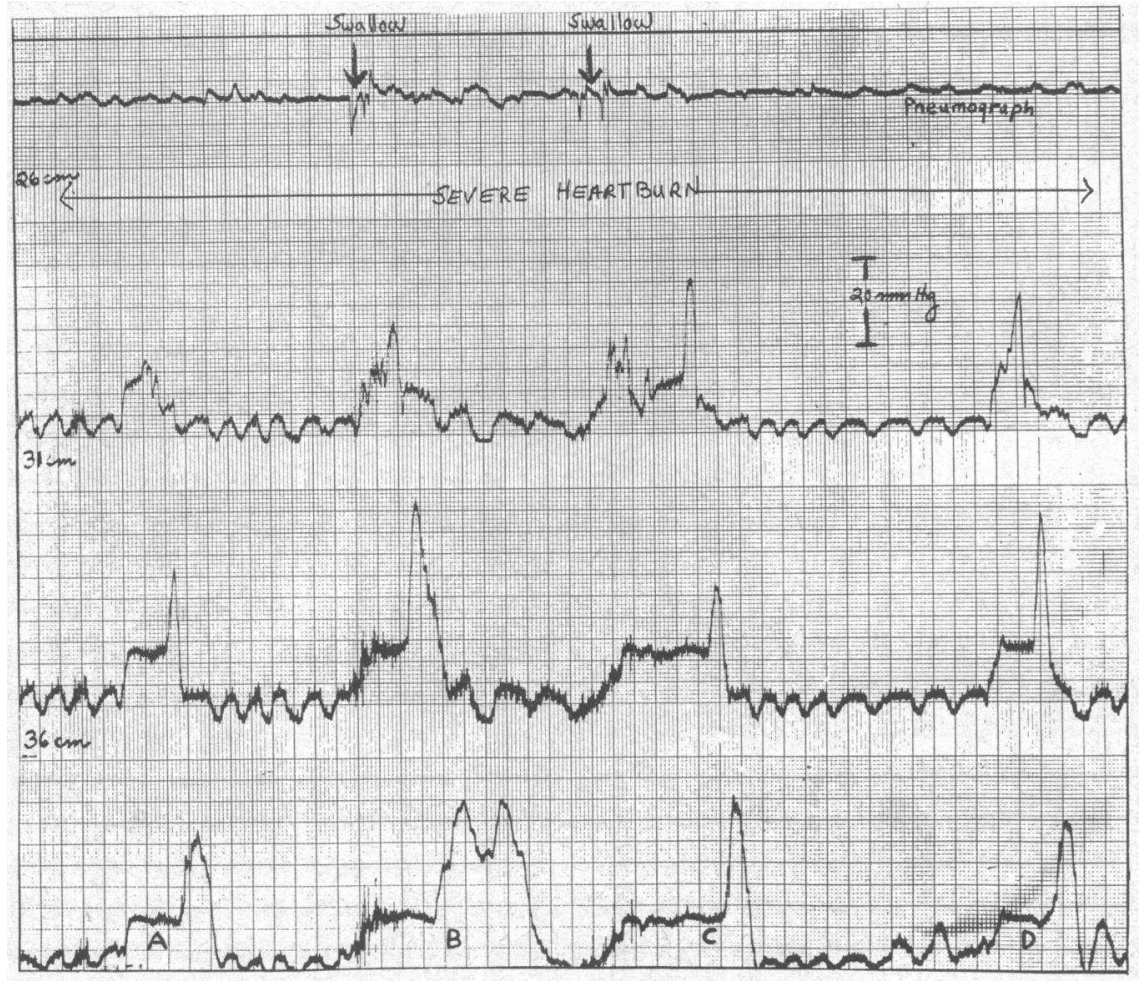

Fig. 6. Electromanometric recordings of Subject 1 (J. L.). The numbers at the left are distances from the incisors. $\mathrm{A}$ and $\mathrm{D}$ are secondary peristaltic responses not preceded by a swallow. $\mathrm{B}$ and $\mathrm{C}$ are primary peristaltic waves after a swallow as the patient attempted to clear acid-tasting material which had refluxed into the throat. 


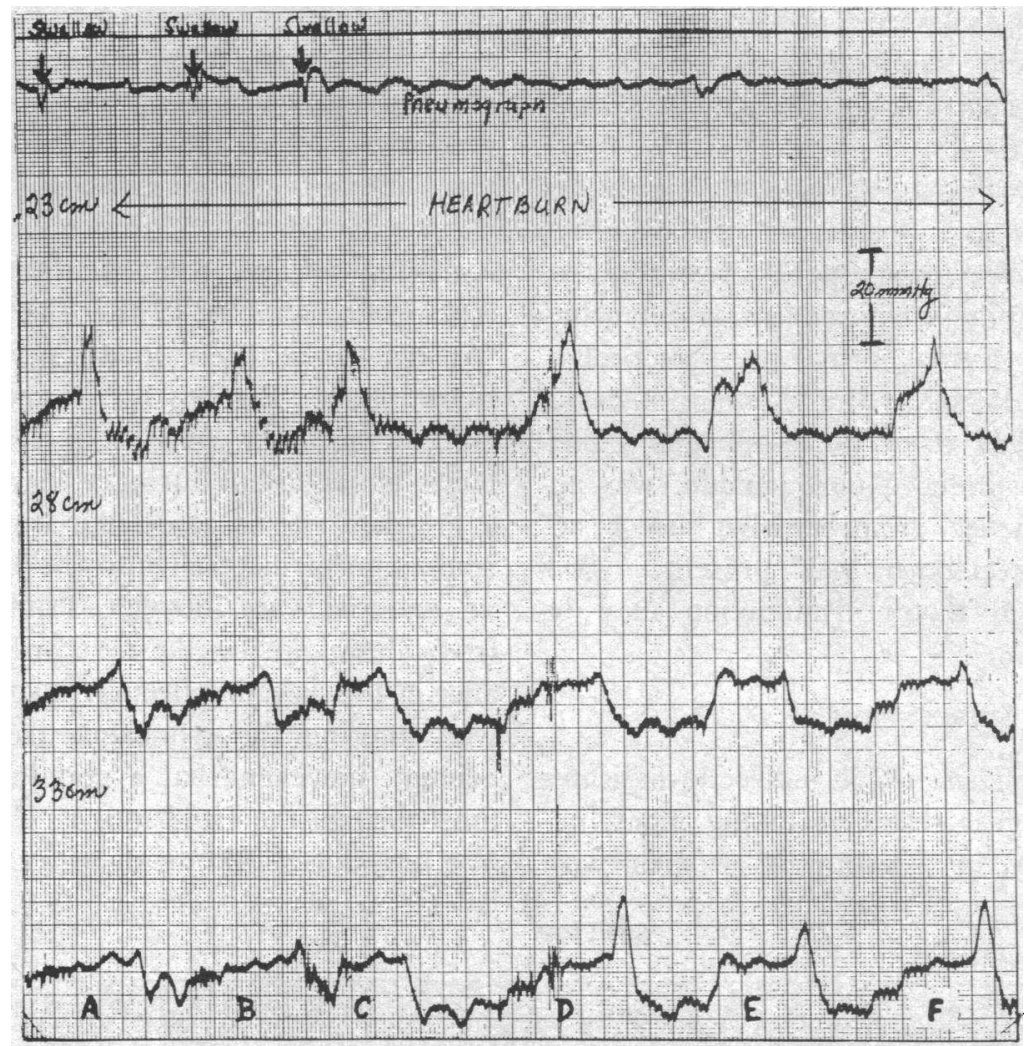

Fig. 7. Electromanometric recordings of Subject 2 (E. H.). The numbers at the left are distances from the incisors. A, B and $\mathrm{C}$ are primary peristaltic responses after a swallow. $\mathrm{D}, \mathrm{E}$ and $\mathrm{F}$ are secondary peristaltic waves not preceded by a swallow. The patient had heartburn throughout this recording.

of mercury secondary peristalsis promptly ensued, but she experienced no heartburn.

Comment. Heartburn occurred during the manometric demonstration of secondary peristalsis in the absence of nonpropulsive motor activity. A similar pressure profile induced post partum was observed without heartburn, suggesting that heartburn does not depend on secondary peristalsis.

Subject 3. H. C., a 38 year old gravida V, had heartburn for the first time in the sixth month of pregnancy. A burning, choking sensation in the throat associated with the occurrence of sourtasting material in the mouth was aggravated by emotional tension and the supine position especially after meals. She never tried antacids, but noted transient relief from drinking a glass of cold water. An esophageal motility study in the ninth month of pregnancy demonstrated secondary peristalsis and prominent nonpropulsive activity not preceded by a swallow. During the first part of the study she complained of heartburn. Immediately after a single swallow of approximately $20 \mathrm{ml}$ of water at room temperature, a normal primary peristaltic wave developed which was promptly followed simultaneously by the sudden cessation of heartburn and an abrupt change in the motility pattern to normal (Figure 8). The fundal esophageal pressure gradient was $5 \mathrm{~mm}$ of mercury. Inferior esophageal sphincter activity occurred only above the effective diaphragmatic hiatus and developed a mean resting end-expiratory pressure of $5 \mathrm{~mm}$ of mercury.

She became asymptomatic after delivery; a follow-up motility study 7 weeks later was normal. The fundal esophageal pressure gradient fell to 2 $\mathrm{mm}$ of mercury, but the mean resting end-expiratory pressure of the inferior esophageal sphincter, however, could now be identified. A pneumatic belt was then placed about her waist and $300 \mathrm{ml}$ of water instilled directly into the stomach. Upon 
inflation of the bag to $100 \mathrm{~mm}$ of mercury, secondary peristalsis and spontaneous nonpropulsive waves promptly occurred, but the patient experienced no heartburn.

Comment. Heartburn was associated with secondary peristalsis and prominent nonpropulsive motor activity during pregnancy. A swallow of water resulted in relief of heartburn simultaneous with the appearance of a normal pressure profile. This might be explained by the offending material having been washed from the esophagus. Secondary peristalsis, induced post partum, was accompanied by similar nonpropulsive motor activity, but no heartburn was present. This suggests that such motor dysfunction may be asymptomatic.

\section{DISCUSSION}

Secondary peristalsis, which indirectly indicates that gastroesophageal reflux is taking place, occurred exclusively in a group of women with heartburn during pregnancy, but in only 45 per cent of the group. Neither heartburn nor secondary peristalsis was observed post partum. What evidence is there that secondary peristalsis indicates reflux? By simultaneous manometric and fluoroscopic studies, Creamer demonstrated an association between secondary peristalsis and gastroesophageal reflux (15). The first pressure deflection seen in the secondary peristaltic complexes in our study (Figures 1, 6, 7 and 8) is characteristic of primary peristalsis following a liquid bolus, but is absent during a "dry" swallow. Such a pressure rise is associated with the sudden introduction of material into the esophagus. Since no material was introduced into the esophagus from above during these recordings, the appearance of this initial deflection suggests that material was entering the esophagus from below. It is evident, however, that a certain amount of fluid must distend the esophagus to elicit this response. We introduced $300 \mathrm{ml}$ of water containing a dye

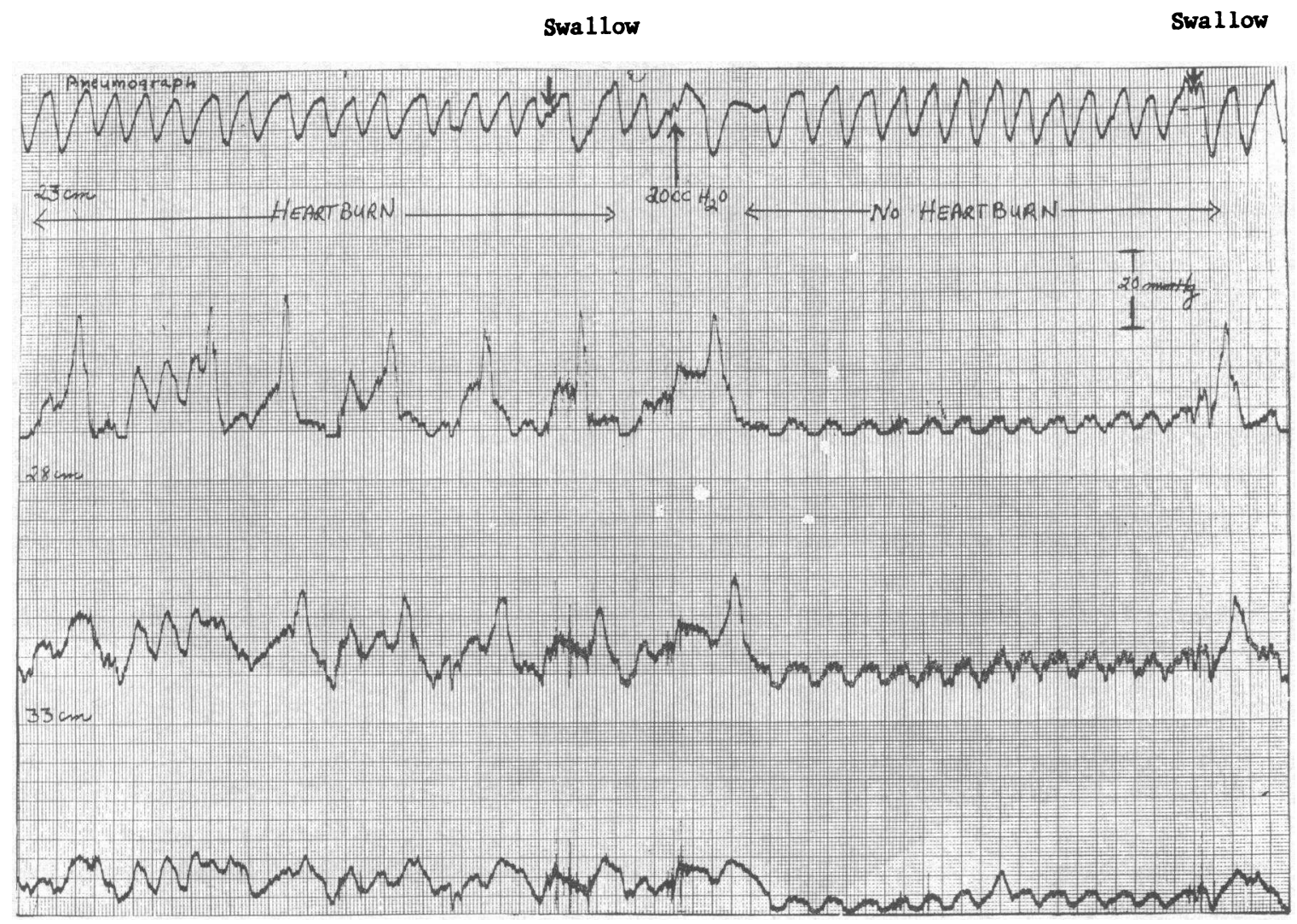

Fig. 8. Electromanometric Recordings of Subject 3 ( H. C.). The numbers at the left are distances from the incisors. The patient experienced heartburn during the appearance of numerous secondary peristaltic waves. After a swallow of $20 \mathrm{ml}$ of water, the heartburn and the secondary peristalsis simultaneously stopped. 
directly into the stomach of 10 control subjects and increased intragastric pressure by inflating a pneumatic abdominal belt. Secondary peristaltic waves appeared in four subjects in whom, at the same time, more than $40 \mathrm{ml}$ of dyed material was aspirated from the distal esophagus. In the other six subjects who did not develop secondary peristaltic waves, less than $10 \mathrm{ml}$ was aspirated from the esophagus, suggesting that the initiation of such a motor response depends upon the regurgitation of relatively large volumes of fluid. We take these results to confirm the relation between secondary peristalsis and gastroesophageal reflux.

The type of material refluxed may bear upon the incidence of heartburn. Reflux occurs in asymptomatic subjects and may be demonstrated during X-ray study of the esophagus and stomach. It is pertinent to note that no symptoms occurred after abdominal pressure was increased by inflation of a pneumatic abdominal belt around the waist in some normal subjects, despite the appearance of secondary peristalsis when $300 \mathrm{ml}$ of water was introduced into the stomach. In contrast, Tuttle, Bettarello and Grossman determined esophageal pressure and intraluminal $\mathrm{pH}$ simultaneously; after $300 \mathrm{ml}$ of $0.1 \mathrm{~N}$ hydrochloric acid had been introduced into the stomach, heartburn occurred when the $\mathrm{pH}$ in the distal esophagus fell below $\mathrm{pH} 4$ (16). In our Patient 1, the reflux of acid appeared, at least from our indirect evidence, to be more important than the mere presence of reflux. Severe heartburn occurred simultaneously with the appearance of secondary peristaltic waves. Although symptoms were relieved with a liquid antacid, the pressure profile remained unchanged. It is true that basal gastric acid is diminished in late pregnancy, and that peptic ulcers are rare during pregnancy $(17,18)$. Nevertheless, whatever acid and peptic material is available may repeatedly gain access to the esophageal mucosa and over a long period of time may produce esophagitis. In animals, acid gastric juice may devastate esophageal mucosa (19).

A number of factors may contribute to reflux. Increased intragastric pressure is the most obvious: the enlarging uterus allows less room for stomach contents. Many pregnant women experience symptoms of reflux when bending or stooping, actions which result in a sharp rise in intragastric pressure (20). Such a rise in pres- sure alone might be responsible for reflux, but while the fundal esophageal pressure gradient increased in most pregnant subjects as the uterus enlarged, the women in whom reflux occurred had no higher intragastric pressure than those without reflux, nor did Creamer find that the fundal esophageal pressure gradient rose during spontaneous reflux in nonpregnant subjects (15). Apparently, elevated intragastric pressure must be associated with diminished resistance of the normal barriers if significant reflux is to occur.

Three mechanisms probably help to prevent gastroesophageal reflux: a direct compression or "pinch-cock" action on the esophagogastric junction exerted by the right rrus of the diaphragm, an intrinsic sphincter mechanism in the distal esophagus, and a passive mucosal valve formed by the acute angle of entry of the esophagus into the stomach $(21,22)$. These three barriers probably reinforce each other when properly aligned. We have studied only the sphincteric mechanism.

The variation in the mean resting end-expiratory pressure of the inferior esophageal sphincter from one individual to another is considered by some observers to represent a technical failure of recording methods $(23,24)$. Serial studies in our control subjects, however, demonstrate that some normal people simply have a well developed intrinsic sphincter mechanism while others do not, but that the tone of the sphincter is relatively constant for the individual. In contrast, in 55 per cent of the pregnant women with heartburn and in 20 per cent of those without heartburn, the resting sphincter pressure decreased with advancing pregnancy and returned to normal levels after delivery. The fall in sphincter pressure may result from gastric atony during pregnancy (25) extending to involve the distal esophagus, which would lower the pressure barrier to reflux. More likely, however, this decrease in resistance to gastroesophageal reflux may be related to loss of the intra-abdominal segment of the sphincter; an intra-abdominal segment was demonstrated in 80 per cent of the controls, but in only 15 per cent of the pregnant women with heartburn and in 37 per cent of those without heartburn. Fyke, Code and Schlegel originally emphasized that an intra-abdominal segment of the sphincter provides an effective barrier to reflux since its intrinsic resistance is supported by the intraperitoneal pres- 
sure (2). Further, it has been graphically demonstrated that when intraperitoneal pressure rises, the pressure in the intra-abdominal segment of the sphincter also rises to preserve the normal pressure gradient (26). The intrathoracic segment does not respond with such an increase in pressure, so that if the sphincter is confined to the thorax, as it is in many pregnant women, the normal pressure barrier may be diminished.

In addition, the acute angle of entry of the esophagus into the stomach, so essential in maintaining the flap valve mechanism, is altered by the displacement of the stomach to a more horizontal position by the enlarging uterus (25), and this barrier to reflux may be lost.

A consideration of factors causing gastroesophageal reflux in pregnancy must include hiatus hernia. Three manometric findings are usually interpreted as indicative of hiatus hernia: continuation of intra-abdominal pressures into the thorax, demonstration of the inferior esophageal sphincter more than $3 \mathrm{~cm}$ above the effective diaphragmatic hiatus and recognition of a "biphasic" point of respiratory reversal $(14,27,28)$. We observed only the latter phenomenon in several women. Since careful roentgenologic examination failed to identify a hiatus hernia in 12 subjects with a biphasic point of respiratory reversal that we have studied, we cannot say with any surety which of the pregnant women had hiatus hernia. Of 195 women in the last trimester of pregnancy, hiatus hernia wás radiographically demonstrated in 18.1 per cent of the multiparae and in 5.1 per cent of the primiparae, a combined incidence of 12.8 per cent (29). No relation to heartburn was evident, but those women who showed reflux often had heartburn (29). Although hiatus hernia occurs more often in multiparae, we found no difference in the incidence of secondary peristalsis between primiparae and multiparae. This suggests that while hiatus hernia may have been a factor in some of our patients, it is not an essential factor.

The passage of a tube into the stomach might be responsible for reflux. We have not found a tube to induce reflux by cineradiographic studies of cardioesophageal competence during shortterm gastric intubation (30).

Although reflux of an acid may initiate heartburn, the immediate pathogenesis of the symptom is controversial. Muscular dysfunction of the esophagus induced either by distention or by acid reflux may be the final pathway resulting in symptoms of heartburn. Nonpropulsive esophageal pressures developed in patients with heartburn induced by continuous intra-esophageal infusion of $0.1 \mathrm{~N}$ hydrochloric acid or equally when heartburn resulted from infusions of other fluids (31). These motor phenomena, however, particularly in the lower two-thirds of the esophagus, are evident in all people, and increase with age (21). Six of 14 asymptomatic young persons whom we have studied demonstrated inconsistently such activity (32). The pregnant women in this study had a marked increase in the frequency and consistency of nonpropulsive responses, but no difference was noted between those women with and without heartburn. The findings in Subjects 2 and 3 are pertinent. Subject 2 demonstrated secondary peristalsis, but nonpropulsive activity did not appear, suggesting that heartburn may occur in its absence. Subject 3 also demonstrated secondary peristalsis, but in addition developed numerous simultaneous nonperistaltic responses representing irritability of the esophagus. When this subject was examined post partum a similar pressure profile was induced but without heartburn, indicating that this motor abnormality may be asymptomatic. Nonpropulsive activity does not appear to be the final common pathway in the pathogenesis of heartburn in pregnancy.

Why pregnant women appear to have an increased frequency of nonpropulsive activity is not clear. Increased intragastric pressure during pregnancy could force air or liquid into the esophagus, which might rapidly transmit pressure along the esophageal lumen so that a pressure response in one area of the organ might be immediately apparent in an adjacent area (33). This is supported by the observation that a normal peristaltic pressure sequence invariably occurred after a swallow of $10 \mathrm{ml}$ of water which could drive air or liquid from the esophagus back into the stomach. In addition, Creamer observed nonpropulsive activity when air was regurgitated into the esophagus (15).

During pregnancy three women with heartburn showed a pressure profile consistent with diffuse esophageal spasm which remained similarly disturbed in the asymptomatic postpartum period. 
This does not support a causal relationship. On the other hand, since this pressure profile was observed only in pregnant women with heartburn, it is possible that the abnormal pattern is an indication of motor derangement that with stimulation becomes more profound and produces symptoms.

\section{SUM MARY}

Serial esophageal motility studies on pregnant women with and without heartburn and on a group of asymptomatic normal young women of similar age indicate that heartburn of late pregnancy is not determined exclusively by motor changes in the esophagus. Nonpropulsive motor activity was seen consistently and with much greater frequency in the pregnant than in the control subjects, but no difference was noted between pregnant women with heartburn and those without heartburn. It seems likely that such activity is related to pregnancy but not to heartburn.

Diffuse esophageal spasm was found in three pregnant women with heartburn. After delivery this abnormal motor pattern persisted, even though symptoms no longer occurred. It is evident that diffuse esophageal spasm is not in itself responsible for heartburn, but since it occurred in pregnant women with heartburn, it may be an associated abnormality.

Acid reflux into the esophagus appears to be a factor in heartburn of late pregnancy. Secondary peristaltic activity suggests that gastric reflux is taking place into the esophagus and the finding of these complexes exclusively in 45 per cent of pregnant women with heartburn provides indirect evidence to support this concept. Gastroesophageal reflux is favored during pregnancy by a combination of factors. Intraperitoneal pressure rises with advancing pregnancy. The inferior esophageal sphincter, which represents a barrier to reflux, develops progressively weaker resting pressures with advancing pregnancy in more than one-half of the pregnant women with heartburn. The intra-abdominal segment of the sphincter could not be identified in the great majority of these women. Maintenance of this segment appears crucial to sustaining the pressure barrier against reflux especially when intraperitoneal pressure is elevated.

\section{ACKNOWLEDGMENTS}

We are grateful to Dr. Massimo Calabresi and his technician, Miss Analee Gelman, for their help in the preliminary stages of this study and to Drs. Clarence D. Davis, Walter Herrmann and Luigi Mastroianni for allowing us to study their patients, and to Mr. Frank Marks and Miss Barbara Van Duzer for their patience and fortitude in the seemingly endless withdrawal of catheters.

\section{REFERENCES}

1. Nagler, R., and Spiro, H. M. A prospective clinical study of heartburn in pregnancy. In preparation.

2. Fyke, F. E., Jr., Code, C. F., and Schlegel, J. F. The gastroesophageal sphincter in healthy human beings. Gastroenterologia (Basel) 1956, 86, 135.

3. Muller Botha, G. S., Astley, R., and Carré, I. J. A combined cineradiographic and manometric study of the gastro-oesophageal junction. Lancet 1957, $1,659$.

4. Sanchez, G. C., Kramer, P., and Ingelfinger, F. J. Motor mechanisms of the esophagus, particularly of its distal portion. Gastroenterology 1953, 25, 321.

5. Fleshler, B., Hendrix, T. R., Kramer, P., and Ingelfinger, F. J. Resistance and reflux function of the lower esophageal sphincter. J. appl. Physiol. 1958, 12, 339.

6. Butin, J. W., Olsen, A. M., Moersch, H. J., and Code, C. F. A study of esophageal pressures in normal persons and patients with cardiospasm. Gastroenterology 1953, 23, 278.

7. Vantrappen, G., Liemer, M. D., Ikeya, J., Texter, E. C., Jr., and Barborka, C. J. Simultaneous fluorocinematography and intraluminal pressure measurements in the study of esophageal motility. Gastroenterology 1958, 35, 592.

8. Hightower, N. C., Jr. Swallowing and esophageal motility. Amer. J. dig. Dis. 1958, 3 (n. s.) 562.

9. Hightower, N. C., Jr. Esophageal motility in health and disease. Dis. Chest 1955, 28, 150.

10. Texter, E. C., Jr., Smith, H. W., Moeller, H. C., and Barborka, C. J. Intraluminal pressures recorded from the upper gastrointestinal tract. I. Correlation with motor activity in normal subjects and patients with esophageal disorders. Gastroenterology 1957, 32, 1013.

11. Fleshler, B., Hendrix, T. R., Kramer, P., and Ingelfinger, F. J. The characteristics and similarity of primary and secondary peristalsis in the esophagus. J. clin. Invest. 1959, 38, 110.

12. Siegel, C. I., and Hendrix, T. R. Evidence for central mediation of secondary peristalsis (abstract). Clin. Res. 1960, 8, 205.

13. Creamer, B., and Schlegel, J. F. Motor responses of the esophagus to distention. J. appl. Physiol. 1957, 10, 498. 
14. Creamer, B., Donaghue, F. E., and Code, C. F. Pattern of esophageal motility in diffuse spasm. Gastroenterology 1958, 34, 782.

15. Creamer, B. Oesophageal reflux. Lancet 1955, 1, 279.

16. Tuttle, S. G., Bettarello, A., and Grossman, M. I. Heartburn and gastroesophageal regurgitation (abstract). Clin. Res. 1960, 8, 100.

17. Spiro, H. M., Schwartz, R. D., and Pilot, M. L. Peptic ulcer in pregnancy. A serial study of gastric secretion during pregnancy. Amer. J. dig. Dis. 1959, 4, 289.

18. Strauss, M. B., and Castle, W. B. Studies of anemia during pregnancy. I. Gastric secretion in pregnancy and the puerperium. Amer. J. med. Sci. 1932, 184, 655.

19. Ferguson, D. J., Sanchez-Palomera, E., Sako, Y., Clatworthy, H. W., Jr., Toon, R. W., and Wangensteen, O. W. Studies on experimental esophagitis. Surgery 1950, 28, 1022.

20. Marchand, $P$. The gastro-oesophageal sphincter and mechanism of regurgitation. Brit J. Surg. 1955, 42, 504 .

21. Ingelfinger, F. J. Esophageal motility. Physiol. Rev. 1958, 38, 533.

22. Vantrappen, G., Texter, E. C., Jr., Barborka, C. J., and Vandenbroucke, $J$. The closing mechanism at the gastroesophageal junction. Amer. J. Med. 1960, 28, 564 .

23. Code, C. F., and Schlegel, J. F. The pressure profile of the gastroesophageal sphincter in man: An improved method of detection. Proc. Mayo Clin. 1958, 33, 406.

24. Pert, J. H., Davidson, M., Almy, T. P., and Sleisenger, M. H. Esophageal catheterization studies. I.
The mechanism of swallowing in normal subjects with particular reference to the vestibule (esophagogastric sphincter). J. clin. Invest. 1959, 38, 397.

25. Williams, N. H. Variable significance of heartburn. Amer. J. Obstet. Gynec. 1941, 42, 814.

26. Nagler, R., and Spiro, H. M. Segmental response of the inferior esophageal sphincter to elevated intragastric pressure. Gastroenterology 1961. In press.

27. Atkinson, M., Edwards, D. A. W., Honour, A. J., and Rowlands, E. N. The oesophagogastric sphincter in hiatus hernia. Lancet 1957, 2, 1138.

28. Texter, E. C., Jr., Lazar, H. P., Puletti, E. J., and Vantrappen, G. The characteristic pattern of esophageal dysfunction due to hiatal hernia demonstrated by fluorocinematography and simultaneous pressure recording (abstract). J. clin. Invest. 1959, 38, 1048.

29. Rigler, L. G., and Eneboe, J. B. The incidence of hiatus hernia in pregnancy and its significance. J. thorac. Surg. 1934, 4, 262.

30. Nagler, R., Wolfsohn, A. W., Lowman, R. M., and Spiro, H. M. The effect of gastric intubation on the normal mechanisms preventing gastro-esophageal reflux. New Engl. J. Med. 1960, 262, 1325.

31. Lazar, H. P., Puletti, E. J., Douglas, W. W., Danovitch, S., and Texter, E. C., Jr. Nonperistaltic esophageal motility accompanying experimentally produced heartburn (abstract). J. Lab. clin. Med. 1959, 54, 917.

32. Nagler, R., and Spiro, H. M. Serial esophageal motility studies in normal young subjects. In preparation.

33. Ingelfinger, F. J. Personal communication. 